\title{
Screening model for nanowire surface-charge sensors in liquid
}

\section{Sørensen, Martin Hedegård; Mortensen, Asger; Brandbyge, Mads}

\section{Published in:}

Applied Physics Letters

Link to article, DOI:

$10.1063 / 1.2779930$

Publication date:

2007

Document Version

Publisher's PDF, also known as Version of record

Link back to DTU Orbit

\section{Citation (APA):}

Sørensen, M. H., Mortensen, A., \& Brandbyge, M. (2007). Screening model for nanowire surface-charge sensors in liquid. Applied Physics Letters, 91(10), 102105. https://doi.org/10.1063/1.2779930

\section{General rights}

Copyright and moral rights for the publications made accessible in the public portal are retained by the authors and/or other copyright owners and it is a condition of accessing publications that users recognise and abide by the legal requirements associated with these rights.

- Users may download and print one copy of any publication from the public portal for the purpose of private study or research.

- You may not further distribute the material or use it for any profit-making activity or commercial gain

- You may freely distribute the URL identifying the publication in the public portal

If you believe that this document breaches copyright please contact us providing details, and we will remove access to the work immediately and investigate your claim. 


\title{
Screening model for nanowire surface-charge sensors in liquid
}

\author{
Martin Hedegård Sørensen, Niels Asger Mortensen, ${ }^{\text {a) }}$ and Mads Brandbyge \\ MIC-Department of Micro and Nanotechnology, Nano $\bullet D T U$, Technical University of Denmark, \\ DTU-Building 345 East, DK-2800 Kongens Lyngby, Denmark
}

(Received 17 July 2007; accepted 14 August 2007; published online 4 September 2007)

\begin{abstract}
The conductance change of nanowire field-effect transistors is considered a highly sensitive probe for surface charge. However, Debye screening of relevant physiological liquid environments challenge device performance due to competing screening from the ionic liquid and nanowire charge carriers. The authors discuss this effect within Thomas-Fermi and Debye-Hückel theory and derive analytical results for cylindrical wires which can be used to estimate the sensitivity of nanowire surface-charge sensors. They study the interplay between the nanowire radius, the Thomas-Fermi and Debye screening lengths, and the length of the functionalization molecules. The analytical results are compared to finite-element calculations on a realistic geometry. (c) 2007 American Institute of Physics. [DOI: 10.1063/1.2779930]
\end{abstract}

Imagine a sensor so small and compact that it can fit almost everywhere and detect all kinds of chemical substances in real time. Such a sensor could, in principle, monitor and detect unwanted bacteria and viruses instantaneously in, e.g., your blood or drinking water. Sensors based on semiconductor nanowires have already been fabricated ${ }^{1}$ and shown to work, for example, as a $p \mathrm{H}$ sensor, where the concentration of hydrogen ions $\mathrm{H}^{+}$in a surrounding liquid is detected. ${ }^{2}$ Moreover, application in label-free detection and biological sensing addressing, e.g., DNA in low concentration is now being explored. ${ }^{3-6}$ In general, the conductance $G$ of a nanowire is in the literature considered a promising candidate for a highly sensitive probe of charged particles covering or situated near its surface. Detection limits have been studied theoretically with respect to binding-diffusion dynamics of the molecules at and near the surface. ${ }^{7,8}$ However, it is also crucial to address the fundamental problem of effective screening of these charges in physiologically relevant liquids which typically yield screening of charges on the nanometer length scale. Of course, it is possible to decrease the salt concentration in the analyte solution, but this may not only complicate the process but also change the biological functions.

Effects of screening have been discussed qualitatively in the literature (see, e.g., Refs. 5 and 9) touching on the influence from the competing screening mechanisms of the electron gas in the nanowire and the dissolved ions in the surrounding electrolyte. Very recently the effect has also been discussed in a computational study of a silicon nanowire field-effect transistor. ${ }^{10}$ In this letter, we develop a simple screening model which exemplifies how the desired screening of surface charge by the nanowire charge carriers may be jeopardized by the contending screening by the electrolyte. We derive analytical results for cylindrical wires which can be used to estimate the sensitivity of a nanowire surfacecharge sensor surrounded by electrolyte. The analytical results are compared to finite-element calculations on a realistic geometry.

A surface-charge density $\sigma_{S}$ will perturb the initial charge-carrier density $n_{0}$ in the nanowire by $\Delta n$, thus chang-



ing the initial conductance $G_{0}$ by $\Delta G$. As an example, a positive surface charge will attract additional electrons from the nanowire contacts (equilibrium reservoirs). From simple conservation of charge we arrive at the following expression for the sensitivity:

$$
\frac{\Delta G}{G_{0}}=\frac{\sigma_{S} P}{e n_{0} A} \Gamma \propto n_{0}^{-2 / 3},
$$

with $\Gamma$ being a dimensionless function, between zero and unity, quantifying the actual sensitivity in the presence of Debye screening in the electrolyte and a finite Thomas-Fermi screening in the nanowire. We show that $\Gamma$ scales with the electron density $n_{0}$ as $n_{0}^{1 / 3}$ in the dilute limit, thus leading to a prediction of a $n_{0}^{-2 / 3}$ dependence, contrasting the intuitively expected $n_{0}^{-1}$ dependence. However, high sensitivity is, of course, still associated with low densities.

Above, $P$ is the perimeter of the nanowire cross section (the fraction supporting the surface charge), $A$ is the crosssectional area, and $e$ is the electron charge. For a cylindrical wire of radius $R$, we have $P / A=2 / R$, thus clearly illustrating the benefit of scaling the wires to the nanoregime. Obviously, screening in the liquid will suppress the sensitivity below the bound given by Eq. (1), and a $n_{0}^{-1}$ dependence (the limit $\Gamma=1)$ can only be expected when the Thomas-Fermi screening in the nanowire is much stronger than the Debye screening in the electrolyte surrounding the nanowire so that changes in the density of the electron gas fully compensates the additional surface charge. In our screening model, we consider the induced electrical potential due to a surfacecharge density $\sigma_{S}$ on the outside of an oxide-covered nanowire (see Fig. 1). For the nanowire we employ the ThomasFermi model (see, e.g., Ref. 11), while for the dilute electrolyte we consider a Debye-Hückel approximation (see, e.g., Ref. 12). With these approximations we arrive at the following linear differential equation for the induced electrical potential $\phi$ :

$$
\nabla^{2} \phi=\left\{\begin{array}{cc}
\lambda_{\mathrm{TF}}^{-2} \phi, & \mathbf{r} \in \Omega_{1} \\
0, & \mathbf{r} \in \Omega_{2} \\
\lambda_{D}^{-2} \phi, & \mathbf{r} \in \Omega_{3} \vee \Omega_{4},
\end{array}\right.
$$

where $\lambda_{\mathrm{TF}}$ is the Thomas-Fermi screening length in the nanowire domain $\Omega_{1}$ and $\lambda_{D}$ is the Debye screening length in the 


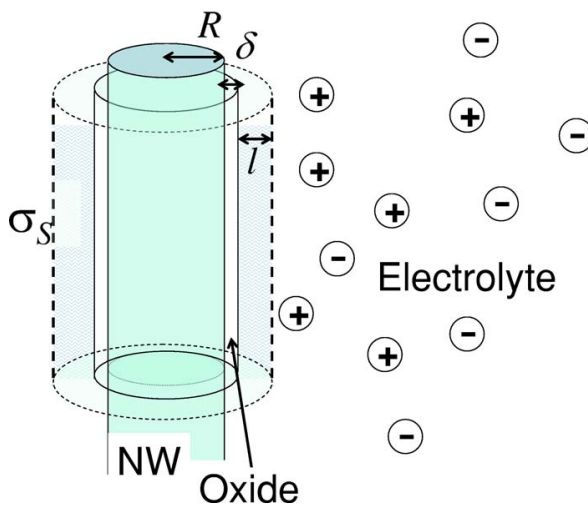

FIG. 1. (Color online) Schematic cross section of a nanowire covered by an ultrathin oxide $(\delta)$ immersed in an electrolyte. A positive surface-charge density $\sigma_{S}$, supported by functionalization molecules of length $\ell$, is screened by negative ions from the electrolyte and electrons from the nanowire. For the opposite sign of $\sigma_{S}$, the surface charge will be screened by attracting positive ions from the electrolyte and by expelling the electrons from the nearby-surface region of the nanowire.

electrolyte domains $\Omega_{3}$ and $\Omega_{4}$. The electrically insulating oxide-layer domain $\Omega_{2}$ is free from charges and the potential is simply a solution to the Laplace equation. Obviously, the induced potential should vanish at infinity, and denoting the solution in domain $\Omega_{i}$ by $\phi_{i}$ and the corresponding dielectric function by $\epsilon_{i}$, we have the additional boundary conditions

$$
\left.\begin{array}{c}
\phi_{i}-\phi_{i+1}=0 \\
\mathbf{n} \cdot \nabla\left[\epsilon_{i} \phi_{i}-\epsilon_{i+1} \phi_{i+1}\right]=\sigma_{i, i+1}
\end{array}\right\}, \quad \mathbf{r} \in \partial \Omega_{i, i+1},
$$

where $\mathbf{n}$ is a normal vector to the surface $\partial \Omega_{i, i+1}$ separating the neighboring domains $\Omega_{i}$ and $\Omega_{i+1}$. Furthermore, $\sigma_{i, i+1}$ is the corresponding surface-charge density. In the following, we consider the case of a surface-charge density $\sigma_{S}$ accumulated at the surface $\partial \Omega_{3,4}$, i.e., $\sigma_{1,2}=\sigma_{2,3}=0$ and $\sigma_{3,4}=\sigma_{S}$. For the dielectric function, we have $\epsilon_{3}=\epsilon_{4}$. The thickness $\ell$ of the domain $\Omega_{3}$ is physically interpreted as the length of the functionalization molecules supporting the charge which for $\ell=0$ resides directly on the outside of the oxide layer of the nanowire. In general, the oxide layer will be charged unless the analyte solution equals the isoelectric point of the surface. Here, we neglect this charge and assume that it may just shift the value of the initial carrier concentration in the wire.

In the following, we consider the conductance $G=G_{0}$ $+\Delta G$ of the nanowire of length $L$ and, in particular, we focus on the conductance change $\Delta G$ due to a finite surfacecharge density $\sigma_{S}$. In terms of the electron mobility $\mu$, we have $G=L^{-1} \int_{\Omega_{1}} d \mathbf{r} e n(\mathbf{r}) \mu$, where $n=n_{0}+\Delta n$ is the electron density and $e$ is the electron charge. We determine the induced charge-density, $n=n_{0}+\Delta n$, from the Poisson equation,

$$
-e \Delta n(\mathbf{r})=-\epsilon_{1} \nabla^{2} \phi_{1}(\mathbf{r})=-\frac{\epsilon_{1}}{\lambda_{\mathrm{TF}}^{2}} \phi_{1}(\mathbf{r})
$$

Formally, the conductance change can now be expressed in terms of the induced potential, i.e.,

$$
\frac{\Delta G}{G_{0}}=\frac{\epsilon_{1}}{\lambda_{\mathrm{TF}}^{2} e n_{0}} \frac{\int_{\Omega_{1}} d \mathbf{r} \phi(\mathbf{r})}{\int_{\Omega_{1}} d \mathbf{r}},
$$

where $G_{0}=\mathrm{Aen}_{0} \mu$ is the conductance in the absence of surface charge. imposed contours show the equipotential lines. As expected,
face charge.
Downloaded 18 Jun 2010 to 192.38.67.112. Redistribution subject to AlP license or copyright; see http://apl.aip.org/apl/copyright.jsp
Equation (4) is our general result for the relative conductance change expressed in terms of an integral over the surface-charge induced potential in the nanowire. Combined with Eqs. (2a) and (2b), it forms a starting point for analytical solutions of simple geometries or numerical solutions of more complicated geometries, e.g., by a finite-element method.

Before turning to more complicated geometries, we first consider the case of a long cylindrical nanowire immersed in an infinite volume of electrolyte (see Fig. 1). The nanowire has a radius $R$ with an oxide layer of thickness $\delta$, and due to the cylinder symmetry, we may solve the problem analytically. For simplicity we focus on the, for experiments, highly relevant limit $\delta \ll R$ of an ultrathin oxide layer. Furthermore, we consider $\ell=0$ so that the charge resides on the outside of the nanowire oxide layer. Solving the linear problem, we then arrive at

$$
\Gamma \simeq\left[1+\frac{\epsilon_{3}}{\epsilon_{1}} \frac{\lambda_{\mathrm{TF}}}{\lambda_{D}} \frac{I_{0}\left(R / \lambda_{\mathrm{TF}}\right) K_{1}\left(R / \lambda_{D}\right)}{I_{1}\left(R / \lambda_{\mathrm{TF}}\right) K_{0}\left(R / \lambda_{D}\right)}\right]^{-1},
$$

which is easily verified to be a function ranging from zero in the limit $\lambda_{D} \ll \lambda_{\mathrm{TF}}$ to unity in the limit $\lambda_{D} \gg \lambda_{\mathrm{TF}}$. We note that in the static limit, water is highly polarizable and $\epsilon_{3} \sim 78 \epsilon_{0}$ while for silicon $\epsilon_{1} \sim 12 \epsilon_{0}$. Thus, for a fixed $n_{0}$ the ultimate sensitivity, Eq. (1) with $\Gamma \sim 1$, requires a very short ThomasFermi screening length, but at the same time one would like to benefit from the $1 / n_{0}$ dependence of $G_{0} \propto n_{0}$. We get $\lambda_{D}=\sqrt{\epsilon_{3} k_{B} T / 2(Z e)^{2} c_{0}}$ in the range of $\lambda_{D} \sim 1-100 \mathrm{~nm}$ at room temperature for ionic concentrations $c_{0}$ for typical physiological electrolytes. For example, ${ }^{10}$ a realistic salt concentration $\left(\mathrm{Na}^{+} \mathrm{Cl}^{-}\right)$of $150 \mathrm{mM}$ yields a screening length of $\lambda_{D} \sim 1 \mathrm{~nm}$, thus calling for nanowires with densities supporting screening at the true nanometer scale. A simple estimate of $\lambda_{\mathrm{TF}}=\sqrt{\hbar^{2} \epsilon_{1} \pi^{4 / 3} / m^{*} e^{2} n_{0}^{1 / 3}}$ also yields $\lambda_{\mathrm{TF}} \sim 1 \mathrm{~nm}$ for a carrier concentration of $10^{18} \mathrm{~cm}^{-3}$. Taylor expanding Eq. (5) in the dilute carrier limit, $\lambda_{\mathrm{TF}} \gg R$, we get

$$
\Gamma \simeq \frac{1}{2} \frac{\epsilon_{1}}{\epsilon_{3}} \frac{\lambda_{D}}{R} \frac{K_{0}\left(R / \lambda_{D}\right)}{K_{1}\left(R / \lambda_{D}\right)}\left(\frac{R}{\lambda_{\mathrm{TF}}}\right)^{2} \propto n_{0}^{1 / 3}
$$

so that we arrive at the $n_{0}^{-2 / 3}$ scaling in Eq. (1).

Figure 2(a) illustrates the dependence of the sensitivity on the Debye screening in the electrolyte. The lower trace is for a nanowire of circular cross section with $\lambda_{\mathrm{TF}} / R=0.02$. The dashed line shows Eq. (5) valid for a negligible oxidelayer thickness, i.e., $\delta \ll R$, while the data points are the result of finite-element simulations (Comsol MultiPhysics) taking into account a finite oxide layer of width $\delta / R=0.005$ and with $\epsilon_{2}=4$. As seen, Eq. (5) accounts well for the numerical exact results. The upper trace shows finite-element results for a nanowire with a trapezoidal cross section with aspect ratio $w / t=2$ and etching defined angle $\theta \sim 54,7^{\circ}$ corresponding to the fabricated structure in Ref. 5 [see Fig. 2(b)]. The oxide layer has a thickness $\delta / t=0.02$, and for the Thomas-Fermi screening, we have $\lambda_{\mathrm{TF}} / t=0.02$, which is somewhat stronger than for the circular case shown in the lower trace. Note how the two curves have the same overall shape and dependence on the Debye screening length, though the stronger ThomasFermi screening for the upper case makes $\Gamma$ approach unity for more moderate Debye screening lengths than in the lower case. Figure 2(b) illustrates a typical distribution of the induced charge-carrier density in the nanowire and the super- 



FIG. 2. (Color online) (a) Sensitivity factor $\Gamma$ vs Debye screening length $\lambda_{D}$. The lower trace: cylindrical nanowire with radius $R$ and $\lambda_{\mathrm{TF}} / R=0.02$. The data points are the result of finite-element simulations with a finite oxidelayer thickness $\delta / R=0.005$. The dashed line shows Eq. (5). Upper trace: finite-element results for the nanowire in (b) shown below. (b) Induced charge-carrier density in a nanowire with trapezoidal cross section and $\lambda_{\mathrm{TF}} / t=0.02$. Aspect ratio $w / t=2$, etching defined angle $\theta \sim 54,7^{\circ}$, and oxide layer $\delta / t=0.02$. Contours show the equipotential lines.

the excess carrier density is induced near the surface of the wire supporting the surface-charge density $\sigma_{S}$.

Finally, let us discuss the prospects for sensing of pointlike charges located at a distance $\ell$ further away from the conductor. Obviously, the additional Debye screening in the layer of thickness $\ell$ (see Fig. 1) will further reduce the induced carrier density in the nanowire, and in a simple picture (neglecting curvature) we would qualitatively expect a reduction proportional to $\exp \left(-\ell / \lambda_{D}\right)$. In the following, we let $N$ denote the average number of molecules absorbed on the wire of length $L$ and we imagine that the chainlike functionalization molecule supports a charged group, with charge $Q$, situated at a distance $\ell$ from the surface. Smearing out these charges results in an equivalent surface-charge density $\sigma_{S}=N Q /[2 \pi(R+\ell) L]$ at $r=R+\ell$. Solving the problem in Eqs. (2a) and (2b) for a finite $\ell$, we get $\Gamma \rightarrow \Gamma_{\ell} \times \Gamma$ with

$$
\Gamma_{\ell} \simeq 2 \frac{R}{R+\ell}\left[1+\sqrt{\frac{R}{R+\ell}} \exp \left(\frac{\ell}{\lambda_{D}}\right)\right]^{-1} .
$$

Here, we have used the large-argument exponential asymptotes for the Bessel functions. The extra factor $0 \leqslant \Gamma_{\ell} \leqslant 1$ illustrates the additional, close to exponential, suppression by Debye screening when the charge is supported by a functionalization molecule of length $\ell$. We note that, in principle, the $\lambda_{D}$ entering the expression for $\Gamma_{\ell}$ could differ from the Debye screening length of the electrolyte, e.g., due to the surface functionalization.

In conclusion, we have used Thomas-Fermi and DebyeHückel theory to formulate a simple screening model for surface-charge sensing with conducting nanowires. The two screening mechanisms act in concert and our model illustrates the nontrivial interplay between the nanowire radius $R$, the Thomas-Fermi screening length $\lambda_{\mathrm{TF}}$, the Debye screening length $\lambda_{D}$, and the length $\ell$ of the functionalization molecules.

The authors thank Jesper Nygård, Brian Skov Sørensen, Per Hedegård, Troels Markussen, and Mogens H. Jakobsen for stimulating discussions.

${ }^{1}$ Y. Cui, Q. Q. Wei, H. K. Park, and C. M. Lieber, Science 293, 1289 (2001).

${ }^{2}$ Y. Chen, X. H. Wang, S. Erramilli, P. Mohanty, and A. Kalinowski, Appl. Phys. Lett. 89, 223512 (2006).

${ }^{3}$ G. F. Zheng, F. Patolsky, Y. Cui, W. U. Wang, and C. M. Lieber, Nat. Biotechnol. 23, 1294 (2005).

${ }^{4}$ W. U. Wang, C. Chen, K. H. Lin, Y. Fang, and C. M. Lieber, Proc. Natl. Acad. Sci. U.S.A. 102, 3208 (2005).

${ }^{5}$ E. Stern, J. F. Klemic, D. A. Routenberg, P. N. Wyrembak, D. B. Turner-Evans, A. D. Hamilton, D. A. Lavan, T. M. Fahmy, and M. A. Reed, Nature (London) 445, 519 (2007).

${ }^{6}$ E. T. Carlen and A. van den Berg, Lab Chip 7, 19 (2007).

${ }^{7}$ P. E. Sheehan and L. J. Whitman, Nano Lett. 5, 803 (2005).

${ }^{8}$ P. R. Nair and M. A. Alam, Appl. Phys. Lett. 88, 233120 (2006).

${ }^{9}$ M. M. C. Cheng, G. Cuda, Y. L. Bunimovich, M. Gaspari, J. R. Heath, H. D. Hill, C. A. Mirkin, A. J. Nijdam, R. Terracciano, T. Thundat, and M. Ferrari, Curr. Opin. Chem. Biol. 10, 11 (2006).

${ }^{10}$ C. Heitzinger and G. Klimeck, J. Comput. Electron. 6, 387 (2007).

${ }^{11}$ N. W. Ashcroft and N. D. Mermin, Solid State Physics (Saunders, San Diego, 1976).

${ }^{12}$ R. Feynman, R. B. Leighton, and M. L. Sands, The Feynman Lectures on Physics (Addison-Wesley, Reading, Massachusetts, 1964). 